\title{
Off the Beaten Path: The Impact of Adaptive Content Sequencing on Student Navigation in an Open Social Student Modeling Interface
}

\author{
Roya Hosseini ${ }^{1}$, I-Han Hsiao ${ }^{2}$, Julio Guerra ${ }^{3}$, and Peter Brusilovsky ${ }^{3}$ \\ 1 Intelligent Systems Program, University of Pittsburgh, Pittsburgh, PA, USA \\ 2 School of Computing, Informatics \& Decision Systems Engineering, Arizona State \\ University, Tempe, AZ, USA \\ 3 School of Information Sciences, University of Pittsburgh, Pittsburgh, PA, USA
}

\begin{abstract}
One of the original goals of intelligent educational systems is to guide every student to the most appropriate educational content. Exploring both knowledge-based and social guidance approaches in past work, we learned that each of these approaches has weak sides. In this paper we follow the idea of combining social guidance with more traditional knowledge-based guidance to support more optimal content navigation. We proposed a greedy sequencing approach that maximizes student's level of knowledge and tested it in a classroom. Results indicated that this approach positively impacts students' navigation.
\end{abstract}

Keywords: personalized guidance, open social student modeling, adaptive navigation support, E-learning, Java programming

\section{Introduction}

One of the original goals of intelligent educational systems was to guide every student to the most appropriate educational content. A range of knowledge-based guidance technologies were reported $[1,6]$. In our recent research we discovered and evaluated a new approach to guide students to the "right" content based on the ideas of open social student modeling (OSSM) [4]. The idea of OSSM is to enhance its cognitive aspects with social aspects by allowing students to explore each other models or cumulative model of the class [2]. Although past studies have shown that OSSM increases student engagement, they also revealed that OSSM approach makes students more conservative in their work with content, which decreases the 'personalization' power of 'social' guidance [4].

This paper explores an idea of combining social guidance with more traditional knowledge-based guidance in a hope to support more optimal content navigation. We introduce a greedy sequencing approach for selecting learning activities that could maximize student's level of knowledge and demonstrate how this approach could be implemented in the context of OSSM. The results of a classroom study confirm the value of sequencing approach in promoting the non-sequential navigation pattern. 


\section{Greedy Sequencing in the Context of OSSM}

To compensate the conforming nature of OSSM on student navigation, we developed a sequencing approach that we call greedy sequencing (GS). The goal of GS is to guide student in the space of learning materials by proactively recommending student activities that could maximize the chance to gain new knowledge while avoiding content that is too complex to comprehend. GS utilizes information about concepts associated with content, more specifically, prerequisite and outcome concepts for each activity. Prerequisite concepts should be mastered before working with the activity while outcome concepts are being learned in the process of working with the activity. All concepts were extracted from contents using our parser [3].

The GS algorithm ranks activities by balancing the knowledge level of the student in the prerequisite concepts and the knowledge that can be gained from the outcome concepts. The rank of an activity (R) is computed using (1):

$$
R=\frac{n_{p} P+n_{o} O}{n_{p}+n_{o}} \quad \text { (1) } P=\frac{\sum_{i}^{n_{p}} k_{i} w_{i}}{\sum_{i}^{n_{p}} w_{i}} \quad(2) \quad O=\frac{\sum_{i}^{n_{o}}\left(1-k_{i}\right) w_{i}}{\sum_{i}^{n_{o}} w_{i}}
$$

where $n_{p}$ and $n_{o}$ are the number of prerequisite and outcome concepts in the activity, respectively; $P$ represents the ratio of known prerequisites and is the weighted average of student's knowledge in the prerequisite concepts of the activity (equation 2); $O$ represents the ratio of unknown outcomes and is the amount not learned in each of the outcome concepts (equation 3). In these two equations $k_{i}$ is the knowledge level of the student in the concept $i$, has the minimum value of 0 and asymtotically reasches 1 . The $w_{i}$ is the smoothed weight of the concept obtained by performing $\log$ function on TF-IDF values of the concepts. The rank $R$ of an activity is in the interval $[0,1]$ with 1 representing the highest rank.

In our study, the GS guidance was implemented inside Mastery Grids, an open social student model interface to access online course materials [5]. Figure 1 shows Mastery Grids. The system organizes a course in topics, displayed as columns of the grid. The first row shows topic-by-topic knowledge progress of the current student by using green colors, the darker the higher the progress. The third row shows the aggregated progress of the rest of the students of the class in shades of orange. The second row presents a differential color comparing the student and the class. For example, in Figure 1 the student has a higher progress than the class in most of the topics where the cells in the second row are green, but the class is more advanced in two of the topics (13th and 20th column) where the cells in the second row are orange. The student has same progress as the class in four topics with light gray color (11th, 15th, 18th, and 19th column). By clicking in cells, the student can access the content inside the topic. For example, in Figure 1, the student has clicked the topic Classes and the system displays cells to access questions and examples related with this topic. Additionally, by clicking the button "Load the rest of learners", an anonymized ranked list of individual student models is shown in a grid form. 
In the current study, we implemented GS on the top of Mastery Grids using red stars to show top -3 recommended items and their containing topics. The size of the star represents the rank of the recommendation. The resulting interface combined the social guidance of OSSM with personal guidance of GS.

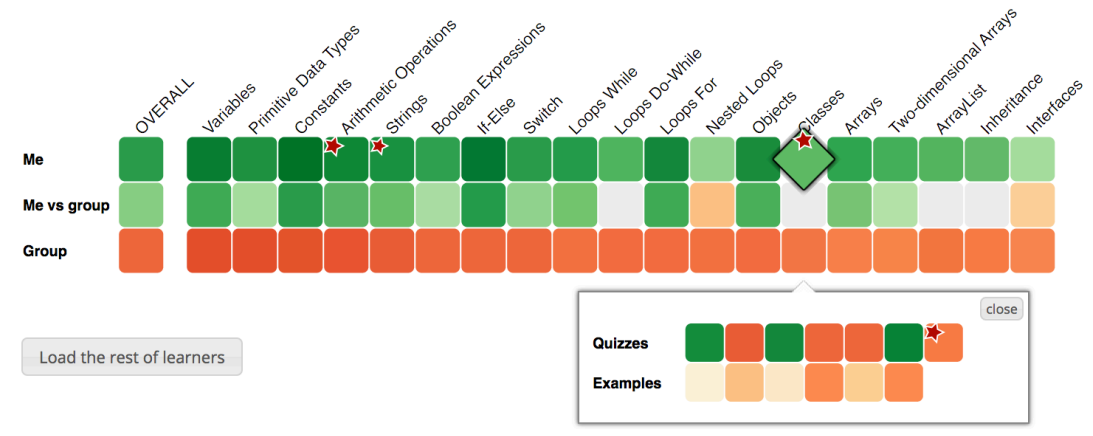

Fig. 1. The presentation of recommendations in the context of Mastery Grids' OSSM interface, a cell with a star symbol represents a recommended topic or activity

\section{Navigational Pattern Analysis}

To explore the effect of GS on students' navigation and performance, we performed a classroom study in an undergraduate programming course offered by the Computer Science Engineering program in Arizona State University during Fall 2014. System usage was non-mandatory. To investigate how students navigate with and without the presence of the sequencing, we split the study into two parts. Part 1, from Aug. 21 to Sep. 25, used Mastery Grids system with no sequencing. In part 2, from Sep. 26 to Oct. 21, sequencing was enabled.

While OSSM interface demonstrated good ability to move the students timely along the common path through the topic sequence, the goal of the GS algorithm was to help the students in breaking out from the common path when it is personally beneficial: not staying too long on already sufficiently mastered topics, while also making sure that knowledge from the past topics are mastered.

To see to what extent the GS encouraged non-sequential navigation, we first classified students' moves from current to next activity into four groups: (1) Within-Topic: moving between activities in the same topic; (2) Next-Topic: moving from an activity in a topic to the activity in the next topic; (3) Jump-Forward: jumping to an activity in a topic two or more steps further; (4) Jump-Backward: jumping to an activity in an earlier topic. Then, we calculated the probability of topic-based patterns across different parts in the study. Figure 2 shows the heatmap frequency table of four navigation patterns in the three contexts: part 1 and part 2 separating student navigation to not-recommended (part 2-N) and recommended activities (part 2-R). The value in each cell is the frequency of the corresponding pattern in each context with light blue for lower probability and dark blue for higher probability. 


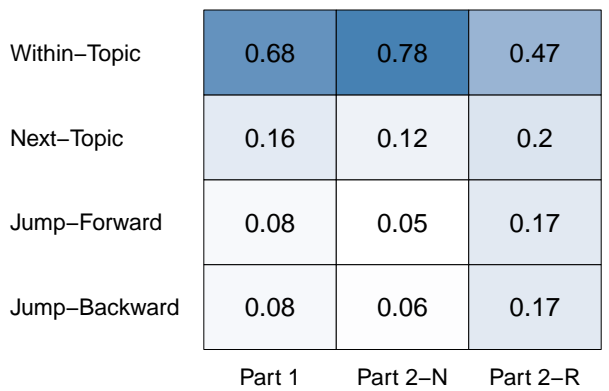

Fig. 2. Relative frequencies of four topic-based navigational patterns during the study

According to this figure, when students make navigation decision without sequencing (Part 1) or ignore it (Part 2-N), they mostly follow sequential pattern working Within-Topic before moving to the Next-Topic. This shows that students tend to attempt most of the activities in the topic before moving to the next one even if it is not the best strategy for their knowledge. The OSSM does hint the students when to move, but its guidance is quite conservative since it is defined by the class as a whole. On the other hand, when students follow GS recommendations, their "groupthink" of staying on the current topic shortens considerably. They move to the next topic faster and remarkably expand their non-sequential navigation (Jump-Forward and Jump-Backward). This is a good evidence that GS encouraged the non-sequential navigation, guiding weaker students to not-mastered materials in previous lectures and advancing stronger to master materials in future lectures. For future work, we plan to investigate the impact of GS recommendations on students' learning.

\section{References}

1. Brecht, B., McCalla, G., Greer, J., Jones, M.: Planning the content of instruction. In: Proceedings of 4-th International Conference on AI and Education, Amsterdam. pp. 24-26 (1989)

2. Bull, S., Britland, M.: Group interaction prompted by a simple assessed open learner model that can be optionally released to peers. In: Proceedings of Workshop on Personalisation in E-Learning Environments at Individual and Group Level (PING), User Modeling. vol. 2007 (2007)

3. Hosseini, R., Brusilovsky, P.: Javaparser: A fine-grain concept indexing tool for java problems. In: The First Workshop on AI-supported Education for Computer Science (AIEDCS 2013). pp. 60-63 (2013)

4. Hsiao, I.H., Bakalov, F., Brusilovsky, P., König-Ries, B.: Progressor: social navigation support through open social student modeling. New Review of Hypermedia and Multimedia 19(2), 112-131 (2013)

5. Loboda, T.D., Guerra, J., Hosseini, R., Brusilovsky, P.: Mastery grids: An open source social educational progress visualization. In: Open Learning and Teaching in Educational Communities, pp. 235-248. Springer (2014)

6. Vassileva, J., Deters, R.: Dynamic courseware generation on the www. British Journal of Educational Technology 29(1), 5-14 (1998) 